\title{
Primary Leiomyosarcoma of the Ovary: A Case Report
}

\author{
Giovanna Rivas ${ }^{1,2,3}$, Carlos Bonilla1,2, Juan Rubiano1,2, Natalia Arango, ${ }^{1,2}$ \\ ${ }^{1}$ Division of Medical Oncology, Cancerology National Institute, Bogotá, Colombia \\ ${ }^{2}$ Universidad el Bosque, Bogotá, Colombia \\ ${ }^{3}$ Integral Cancer Center, Clínica de Occidente, Cali, Colombia \\ Email: govariv@hotmail.com
}

Received 16 February 2014; revised 14 March 2014; accepted 2 April 2014

Copyright (C) 2014 by authors and Scientific Research Publishing Inc.

This work is licensed under the Creative Commons Attribution International License (CC BY).

http://creativecommons.org/licenses/by/4.0/

(c) (i) Open Access

\begin{abstract}
Leiomyosarcoma of the ovary is a rare disease, with aggressive behavior and poor prognosis, which mainly occurs in postmenopausal women. Surgery is the cornerstone of treatment, while the role of chemotherapy and radiotherapy is still not clear because substantial data is lacking. This paper presents the case of an adult woman who is diagnosed with primary leiomyosarcoma of the left ovary, and who receives a surgical approach and adjuvant treatment with chemotherapy currently achieving $\mathbf{2 0}$ months free from disease.
\end{abstract}

\section{Keywords}

Leiomyosarcoma, Ovarian Neoplasms, Treatment

\section{Introduction}

Primary sarcomas of the ovary are rare gynecologic tumors which represent about 4\% of all ovarian tumors [1]. Chondrosarcomas, fibrosarcomas, endometrial stromal sarcomas, angiosarcomas, rhabdomyosarcomas and leiomyosarcomas have been reported [1] [2].

Primary leiomyosarcoma of the ovary represents only 1\% of ovarian tumors; about 60 cases have been reported in the literature [3]. These occur generally in women older than 60 years old, they are frequently diagnosed in advanced stages, they have an aggressive behavior and prognosis is poor, most patients die within the first year of diagnosis [1]-[3].

The cornerstone of treatment is surgery. Benefit from radiotherapy and adjuvant chemotherapy is not clear [1]-[5].

${ }^{*}$ Corresponding author. 
This case is the first literature report on primary leiomyosarcoma of the ovary in which adjuvant treatment with ifosfamide and doxorubicin has been used, achieving progression-free survival at 24 months, and overall survival at 36 months.

\section{Case Description}

A 65 years old woman, with a history of diabetes mellitus, arterial hypertension and hypothyroidism, presented with 18 months of colic abdominal pain in the umbilical and suprapubic region, associated with rectal tenesmus and episodes of urinary retention. She arrived at Hospital de San José in Bogota because of an increase in intensity and frequency of symptoms in April, 2010. An abdominal ultrasound showed a rounded pelvic mass of $97 \times$ $98 \mathrm{~mm}$ that compressed the left inferior wall of the bladder; a transvaginal pelvic ultrasound showed an uterus in anteflexion of $6 \times 4 \mathrm{cms}$, endometrium of $4 \mathrm{~mm}$, and a solid pelvic mass of $11 \times 8 \mathrm{cms}$ which formed a body with the lateral wall of the uterus, the right ovary was normal. An axial computed tomography of the abdomen and the pelvis performed on July 10, 2010, showed a solid pelvic mass with no calcifications and lobed outlines of $14 \times 13 \times 10 \mathrm{cms}$ in close contact with the left adnexal region and the anterior wall of the uterus. A primary tumor of the ovary vs. a subserous myoma were considered possible diagnoses (Figure 1). CA-125 and carcinoembryonic antigen levels were normal.

The patient was scheduled for surgery on September 2010 for hysterectomy, bilateral salpingo oophorectomy, pelvic radical lymphadenectomy and pelvic lavage. During surgery a $15 \times 14 \times 11 \mathrm{cms}$ tumor was found which depended on the left ovary, without apparent compromise of uterus and parametria. Pathology examination reported a tumor lesion in the left ovary which compromised the capsule, formed by fusiform cells set in a random pattern where frequent pleomorphic nuclei are recognized, hyperchromatic, with prominent nuclei, atypical mytoses with counts over 5 in 10 high power fields and necrosis compatible with low grade leiomyosarcoma with negative borders (Figure 2). Fallopian tubes, right ovary, cervix and omentum were negative for tumor lesions.

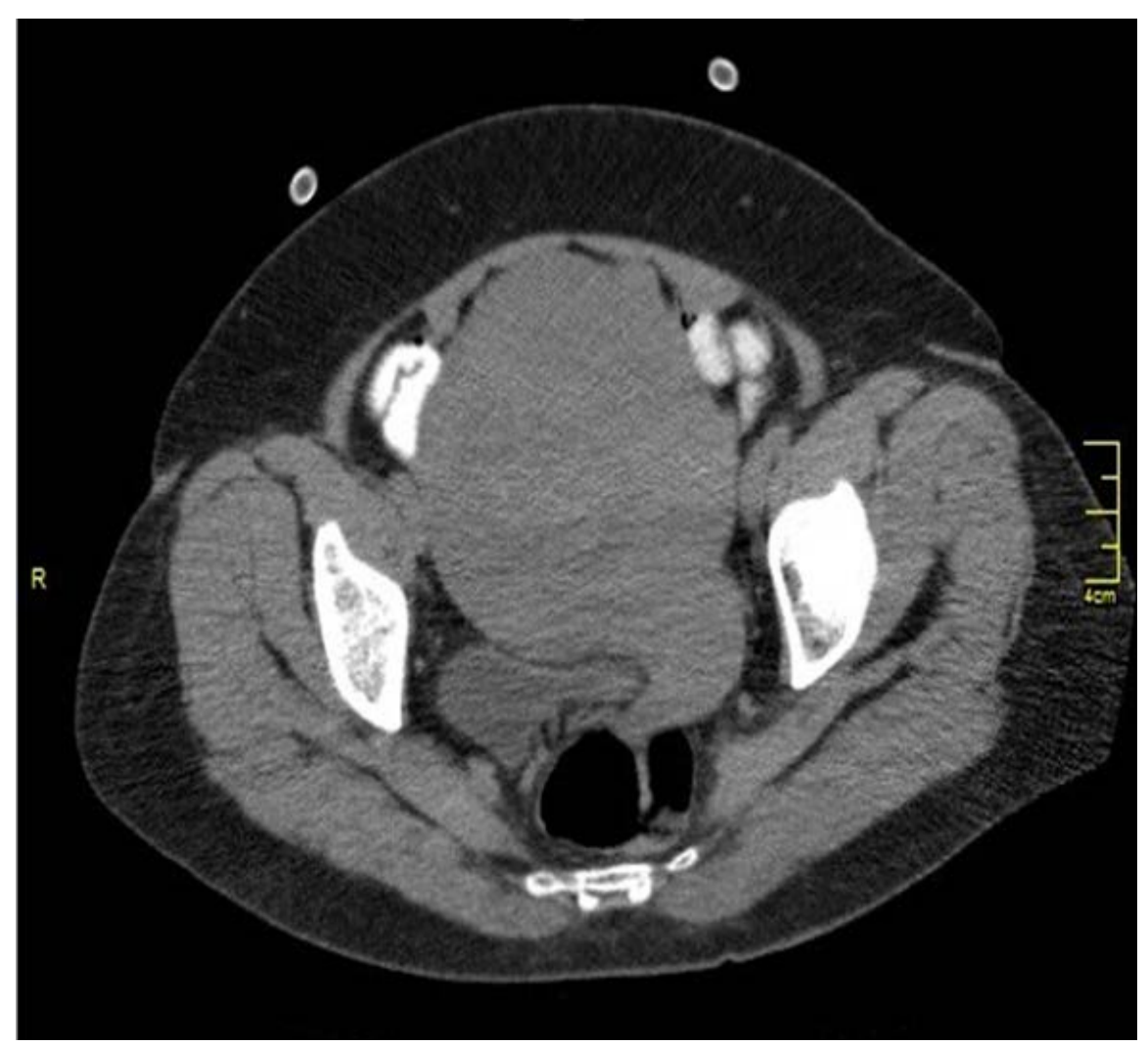

Figure 1. Abdomen and pelvis computed tomography scan initial, show a tumor a solid pelvic mass with no calcifications and lobed outlines of $14 \times 13 \times 10 \mathrm{cms}$ in close contact with the left adnexal region and the anterior wall of the uterus. 

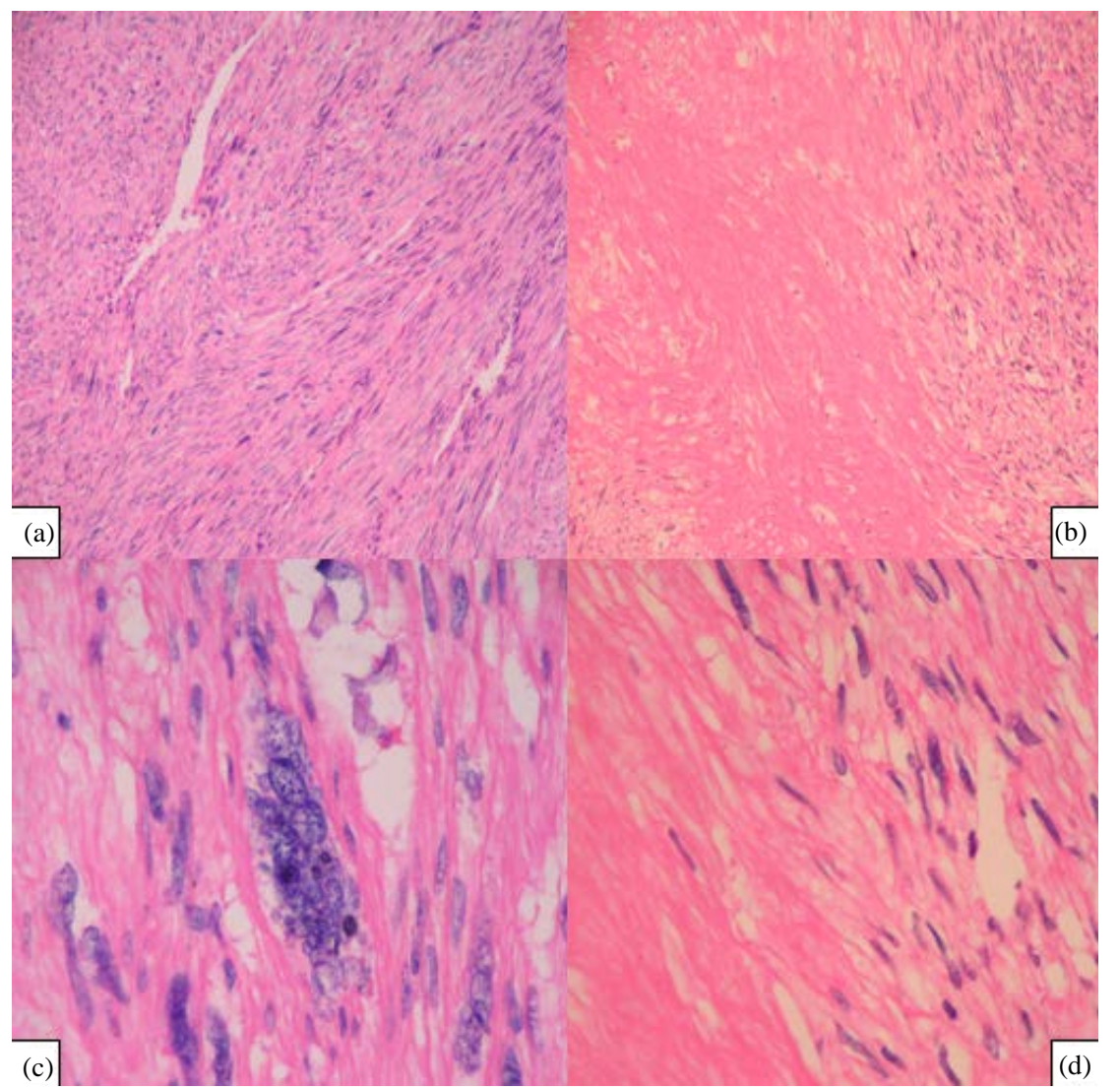

Figure 2. Histopathologic assessment revealed an ovarian tumor, formed by fusiform cells set in a random pattern where frequent pleomorphic nuclei are recognized, hyperchromatic, with prominent nuclei and atypical mytoses with counts over 5 in 10 high power fields and necrosis (Panel (a), (b) Hematoxylin-eosin stain $\times 10$ magnification. Panel (c), (d) Hematoxylin-eosin stain $\times 40$ magnification).

Eight lymphatic ganglia were free of tumor compromise and pelvic and paracolic gutter lavage cytology were negative for malignancy. A diagnosis of stage FIGO IC primary leiomyosarcoma of the left ovary was done.

The patient was referred to continue treatment at Cancerology National Institute. The pathology report showed compromise with leiomyosarcoma grade I-II of the left ovary, immunohistochemistry revealed positivity for smooth muscle actin, caldesmon, vimentin, desmin and progesterone receptors $(<5 \%)$, with proliferation index ki67: 30\% (Figure 3). Chest tomography and abdominal resonance imaging were negative for residual tumor and for metastases.

Adjuvant chemotherapy was offered with doxorubicin $20 \mathrm{mg} / \mathrm{m}^{2}$ on days $1-3$, ifosfamide $2 \mathrm{~g} / \mathrm{m}^{2}$ on days 1 3 and mesna (MAI regimen), she received four cycles without dose limiting toxicity between December 2010 and March 2011. The department of radiotherapy considered that she would not benefit from radiation. The patient is currently asymptomatic and without signs of disease relapse after 24 months of follow-up.

\section{Discussion}

Primary leiomyosarcomas of the ovary are infrequent tumors that represent about $1 \%$ of all malignant tumors of the ovary, they are usually unilateral and occur in women older than 60 years old [1]-[3]; however, some cases have been reported in young patients [3] [4] [6].

Primary leiomyosarcomas of the ovary have a macroscopic appearance of pale, lobed masses with a solid component and occasional cystic degeneration [1] [3]. At microscopic examination fusiform cells predominate and these are arranged vertically with elongated nuclei and eosinophilic cytoplasm [1] [3], other characteristic features are: hypercellularity, cellular atypia, pleomorfism, high mitotic rate (larger than 10 in 10 high power 


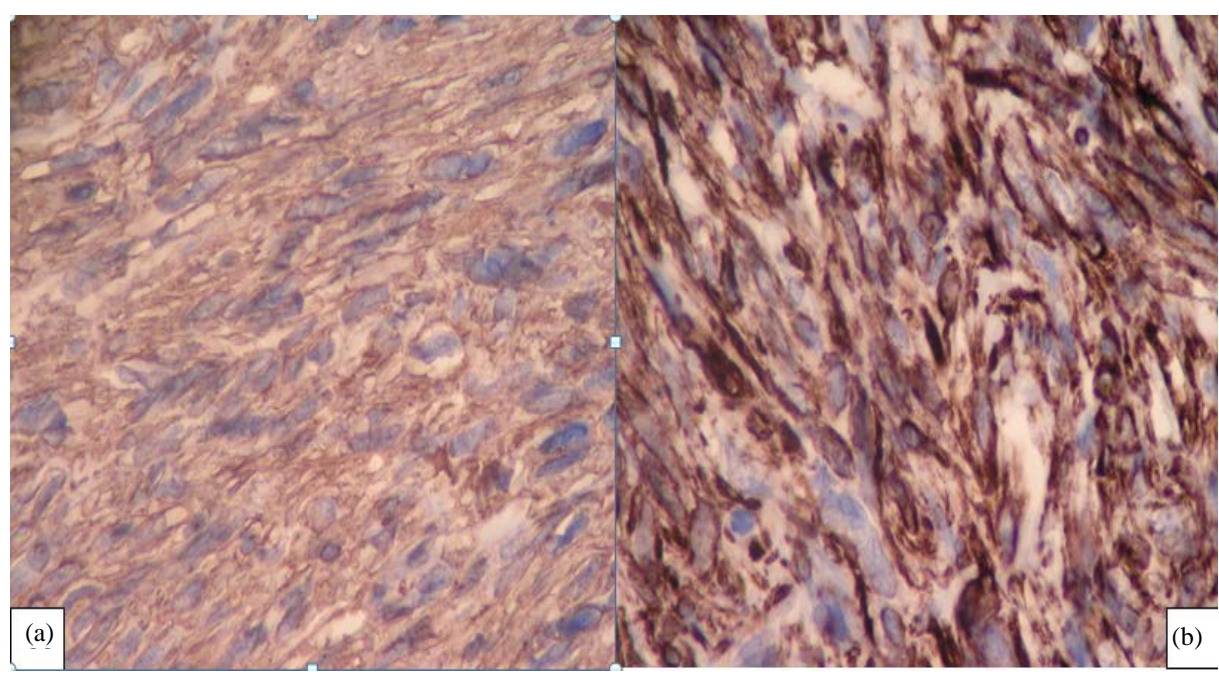

Figure 3. Immunohistochemical staining of the tumor. Panel (a): vimentin stain $\times 40$ magnification. Panel (b): actin stain $\times 40$ magnification.

fields) and coagulation necrosis; they have reactivity for desmin, vimentin and actin of smooth muscle, and being negative for cytokeratins and S100 [1]-[3]. Immunochemistry frequently shows positivity for desmin, vimentin, smooth muscle actin, negative cytokeratins and S100 [1]-[3]. Positivity for estrogen, progesterone, p53 and bcl-2 have been reported in some cases [2] [3].

The pathogenesis is uncertain, an origin from the smooth muscle of the blood vessels of the cortical stroma has been suggested, as well as from the muscles around the corpus luteum and ovarian follicules, muscle fibers attached to the ovarian ligament, the remainder of Wolff's duct, totipotent stem cells of the ovary, teratoma cells that have differenciated or in smooth muscle cells that migrate from within the uterus [2] [3] [6].

Patients frequently present with pain secondary to compression over adjacent organs and a palpable abdominal mass. Many ovarian leiomyosarcomas are diagnosed at advanced stages so that these tumors usually measure larger than $10 \mathrm{~cm}$ [1]-[3]. Early relapses are frequent during the first year of follow-up, usually to pelvis or abdomen [3]-[6], and less commonly these early relapses occur within lung, bone, liver, mediastinum, cerebrum, cervical ganglia and skull [3].

Differential diagnosis of primary leiomyosarcomas of the ovary include leiomyomas, fibrothecomas, fibrosarcomas, endometrial stromal sarcomas, mixed mullerian tumors, fusiform cell carcinoma and gastrointestinal stromal tumor metastases (GIST) [2] [5].

For staging purposes the classification system from International Federation of Gynecology and Obstetrics (FIGO) for ovarian carcinomas is recommended [2] [3].

The cornerstone of treatment is surgery, which has variable extension ranging from fertility-conservative surgery to radical surgery consisting of total abdominal hysterectomy, bilateral oophorectomy, pelvic lymphadenectomy and omentectomy [1]-[3] [5] [7]. Rasmussen et al. described the usefulness of performing cytoreduction surgery in repeated opportunities during relapses and they also suggested that primary leiomyosarcomas of the ovary could be hormone-sensitive [8]. In tumors confined to the ovary some authors have described that surgery could be enough as sole treatment [4].

Neoadjuvant chemotherapy may increase large tumor removability [1]. Benefit from radiotherapy and adjuvant chemotherapy is not clear due to the low number of reported cases [3]-[5]. However, postsurgical radiotherapy may aide in achieving local control and chemotherapy has been recommended for stages II up to IV [1]-[3] where in most cases ifosfamide-based regimens have been used [3] [8]; cisplatin-based regimens have also been described [9].

This case is the first literature report on primary leiomyosarcoma of the ovary in which adjuvant treatment with ifosfamide and doxorubicin has been used, achieving progression-free survival at 24 months, and overall survival at 36 months. Up to now, other regimens for leiomyosarcoma (mainly uterine) has been described as docetaxel plus gemcitabine, trabectidine or pazopanib. In contrast, numerous drugs are clearly ineffective for example temozolomide, topotecan and gefitinib [10]. 
The prognosis of this disease is poor and depends on tumor size, differentiation, mitotic rate and stage; the latter being the most important factor [2] [3] [11]. While 63.3\% of patients in stage I are alive without evidence of disease at 42 months, $81 \%$ of patients in stages II-IV die after 14 months of follow-up [3]. Total surgical resections have also been described to result in good prognosis [9].

We recommend individualizing medical treatment in accordance with patient characteristics and known prognostics factors for sarcomas in other locations.

\section{Conclusion}

Primary leiomyosarcoma of the ovary is a rare incidental disease, with few literature reports, primarily affecting postmenopausal women and generally presenting at an advanced stage. Treatment is based on a surgical approach, while the role of complementary treatment such as chemotherapy and radiotherapy is not clear owing to a lack of data, therefore individual patient background should guide their use with explanation of both risk profiles and treatment benefits. Prognosis is usually poor, but early diagnosis and the surgical staging and optimal cytoreduction may improve survival.

\section{Acknowledgements}

The authors would like to thank Dr. María Islena Beltrán and Dr. Jorge Mesa from the Department of Pathology at Cancerology National Institute, Bogotá-Colombia.

\section{Conflicts of Interest Statement}

The authors declare no conflicts of interest.

\section{References}

[1] Dixit, S., Singhal, S., Baboo, H.A., Vyas, R.K., Neema, J.P., Murthy, R. and Sooryanaraya, U. (1993) Leiomyosarcoma of the Ovary. Journal of Postgraduate Medicine, 39,151-153.

[2] Bouie, S.M., Cracchiolo, B. and Heller, D. (2005) Epithelioid Leiomyosarcoma of the Ovary. Gynecologic Oncology, 97, 697-699. http://dx.doi.org/10.1016/j.ygyno.2005.02.008

[3] Taşkin, S., Taşkin, E.A., Uzüm, N., Ataoğlu, O. and Ortaç, F. (2007) Primary Ovarian Leiomyosarcoma: A Review of the Clinical and Immunohistochemical Features of the Rare Tumor. Obstetrical \& Gynecological Survey, 62, 480-486. http://dx.doi.org/10.1097/01.ogx.0000268629.16151.71

[4] Monk, B.J., Nieberg, R. and Berek, J.S. (1993) Primary Leiomyosarcoma of the Ovary in a Perimenarchal Female. Gynecologic Oncology, 48, 389-393. http://dx.doi.org/10.1006/gyno.1993.1067

[5] Arslan, O.S., Sumer, C., Cihangiroglu, G., Kanat-Pektas, M. and Gungor, T. (2011) A Rare Tumor of the Female Genital Tract: Primary Ovarian Leiomyosarcoma. Archives of Gynecology and Obstetrics, 283, 83-85. http://dx.doi.org/10.1007/s00404-010-1735-3

[6] Azoury, R.S. and Woodruff, J.D. (1971) Primary Ovarian Sarcomas. Report of 43 Cases from the Emil Novak Ovarian Tumor Registry. Obstetrics \& Gynecology, 37, 920-941.

[7] Inoue, J., Gomibuchi, H. and Minoura, S. (2000) A Case of a Primary Ovarian Leiomyosarcoma. Journal of Obstetrics and Gynaecology Research, 26, 401-407. http://dx.doi.org/10.1111/j.1447-0756.2000.tb01349.x

[8] Rasmussen, C.C., Skilling, J.S., Sorosky, J.I., Lager, D.J. and Buller, R.E. (1997) Stage IIIC Ovarian Leiomyosarcoma in a Premenopausal Woman with Multiple Recurrences: Prolonged Survival with Surgical Therapy. Gynecologic Oncology, 66, 519-525. http://dx.doi.org/10.1006/gyno.1997.4824

[9] Dai, Y., Shen, K., Lang, J.H., et al. (2011) Primary Sarcoma or the Ovary: Clinicopathological Characteristics, Prognostic Factors and Evaluation of Therapy. Chinese Medical Journal, 124, 1316-1321.

[10] Penel, N., Van Glabbeke, M., Marreaud, S., Ouali, M., Blay, J.Y. and Hohenberger, P. (2011) Testing New Regimens in Patients with Advanced Soft Tissue Sarcoma: Analysis of Publications from the Last 10 Years. Annals of Oncology, 22, 1266-1272. http://dx.doi.org/10.1093/annonc/mdq608

[11] Nasu, M., Inoue, J., Matsui, M., Minoura, S. and Matsubara, O. (2000) Ovarian Leiomyosarcoma: An Autopsy Case Report. Pathology International, 50,162-165. http://dx.doi.org/10.1046/j.1440-1827.2000.01020.x 